1 Hacettepe Journal of Mathematics and Statistics

$\bigcap$ Volume 45 (6) (2016), 1755-1766

\title{
Approximations in a hyperlattice by using set-valued homomorphisms
}

\author{
Ş. Yılmaz ${ }^{* \dagger}$ and O. Kazanc1 ${ }^{\ddagger}$
}

\begin{abstract}
In this paper, the concepts of set-valued homomorphism and strong setvalued homomorphism of a hyperlattice are introduced. The notions of generalized lower and upper approximation operators constructed by means of a set-valued mapping are provided. We also propose the notions of generalized lower and upper approximations with respect to a hyperideal of a hyperlattice which is an extended notion of rough hyperideal in a hyperlattice and discuss some significiant properties of them.
\end{abstract}

Keywords: Hyperlattice; Hypercongruence; Approximation space; Rough set; Lower and upper approximations; Set-valued mapping.

2000 AMS Classification: 06B75; 06B99.

Received : 16.11.2015 Accepted : 25.01.2016 Doi : 10.15672/HJMS.20164515995

\section{Introduction}

The theory of algebraic hyperstructures is a well-established branch of classical algebraic theory which were initiated by Marty [15]. In a classical algebraic structure, the composition of two elements is an element while in an algebraic hyperstructure the composition of two elements is a set. Hundreds of papers and several books have been written on hyperstructure theory, see for instance [5,6]. Hyperlattices were first studied by Konstantinidou and Mittas [18]. Since the concept of hyperlattice is a generalization of the concept of lattice, hyperlattice theory was studied by Konstantinidou [19-21], Ashrafi [3], Rahnamai-Barghi [29-30] Guo and Xin [14], Han and Zhao [12], Zhao and Han [37].

Rough set theory was proposed by Pawlak [26]; see also [27-28]. The theory of rough sets is an extension of set theory, in which a subset of a universe is described by a pair of

*Department of Mathematics, Karadeniz Technical University,

61080, Trabzon, Turkey, Email: serifeyilmaz@ktu.edu.tr

$\dagger$ Corresponding Author.

‡Department of Mathematics, Karadeniz Technical University, 61080, Trabzon, Turkey, Email: kazancio@yahoo.com 
ordinary sets called the lower and upper approximations. A key concept in Pawlak rough set model is the equivalence relation. The equivalence classes are the building blocks for the construction of the lower and upper approximations. However, the requirement of an equivalence relation in Pawlak rough set model seems to be a very restrictive condition that may limit the applications of rough set models. Thus, one of the main directions of research in rough set theory is naturally the generalization of Pawlak rough set approximations. For instance, the notion of approximations are extended to general binary relations, coverings, completely distributive lattices, fuzzy lattices and Boolean algebras. This research soon led to a natural question concerning the possible connection between rough sets and algebraic systems.

In [22], Kuroki introduced a rough ideal in a semigroup. Kuroki and Wang [23] presented some properties of the lower and upper approximations with respect to normal subgroups. Davvaz [8] investigated the relationship between rough sets and ring theory by considering a ring as a universal set and introducing the concepts of rough subrings and rough ideals with respect to an ideal of a ring. Kazanc1 and Davvaz [16] introduced the notions of rough prime (primary) ideals and rough fuzzy prime (primary) ideals in a ring and presented some properties of such ideals. Rough semigroups, rough modules, rough lattices, rough $\mathrm{MV}$-algebras, rough hemirings and rough $\gamma$ semihyperrings have been investigated by many authors( see also [1,2,4,7,8,11,17,19,24,25,31,34]). Davvaz and Mahdavipour [10] presented a framework for generalizing the standard notion of rough set approximation space. They proposed new definitions of the lower and upper approximations which are basic concepts of rough set theory. In [9], Davvaz introduced the concept of set-valued homomorphism for groups which is a generalization of an ordinary homomorphism. The concepts of set-valued homomorphism and strong set-valued homomorphism of a ring were introduced by Yamak et al.[35] and Hooshmandasl et al. [13].

The initiation and majority of studies on rough sets for algebraic structures have been concentrated on a congruence relation. The congruence relation, however, seems to restrict the application of the generalized rough set model for algebraic sets. This may be by reason of incomplete information about the objects under consideration. Sometimes due to imprecise human knowledge about the elements of the universe set, an equivalence relation among these elements is difficult to find. To overcome this problem, we require set-valued maps instead of equivalence relations in generalized rough sets. This technique is useful where it is not easy to find a equivalence relation among the objects of the universe set. This paper is structured as follows. After an introduction, in Section 2, we present some basic definitions and results about approximation operators. In Section 3, we restrict the universe of the approximation space to a hyperlattice and we introduce the axiomatic form of this concept. In Section 4, the concepts of generalized lower and upper approximation operators constructed by means of a set-valued homomorphism with respect to a hyperideal of a hyperlattice is presented and we examine some properties of these operators in a hyperlattice.

\section{Preliminaries}

In this section, we recall some notions and results (see $[5,6,14,15,20]$ ) which will be used throughout this article. Let $L$ be a non-empty set and $P^{*}(L)$ be the set of all nonempty subsets of $L$. A hyperoperation on $L$ is a map $\circ: L \times L \rightarrow P^{*}(L)$ which associates a nonempty subset $a \circ b$ with any pair $(a, b)$ of elements of $L \times L$. The couple $(L, \circ)$ is called a hypergroupoid. If $A$ and $B$ are nonempty subsets of $L$, then for $a, b, x \in L$, we denote 
(1) $x \circ A=\{x\} \circ A=\bigcup_{a \in A} x \circ a, A \circ x=A \circ\{x\}=\bigcup_{a \in A} a \circ x$. (2) $A \circ B=\bigcup_{a \in A, b \in B} a \circ b$.

2.1. Definition. [14] Let L be a non-empty set endowed with two hyperoperations $\otimes$ and $\oplus$. The triple $(L, \otimes, \oplus)$ is called a hyperlattice if the following conditions hold for all $a, b, c \in L$ :

(1) (idempotent laws) $a \in a \otimes a, a \in a \oplus a$,

(2) (commutative laws) $a \otimes b=b \otimes a, a \oplus b=b \oplus a$,

(3) (associative laws) $(a \otimes b) \otimes c=a \otimes(b \otimes c),(a \oplus b) \oplus c=a \oplus(b \oplus c)$,

(4) (absorption laws) $a \in a \otimes(a \oplus b), a \in a \oplus(a \otimes b)$.

2.2. Definition. [14] Let $L=(L, \otimes, \oplus)$ be a hyperlattice and $S \in P^{*}(L)$. Then $S$ is called a subhyperlattice of $L$ if $a \otimes b$ and $a \oplus b \in P^{*}(S)$ for all $a, b \in S$. That is to say, $S$ is subhyperlattice of $L$ if and only if $S$ is closed under the two hyperoperation $\otimes$ and $\oplus$ on $L$.

2.3. Example. Let $L=\{a, b, c, d\}$ be a set. Define the hyperoperations " $\otimes$ " and ${ }^{\prime \prime} \oplus$ " on $L$ with the following Cayley table:

\begin{tabular}{c|cccc}
$\otimes$ & $\mathrm{a}$ & $\mathrm{b}$ & $\mathrm{c}$ & $\mathrm{d}$ \\
\hline $\mathrm{a}$ & $\mathrm{a}$ & $\mathrm{a}$ & $\mathrm{a}$ & $\mathrm{a}$ \\
$\mathrm{b}$ & $\mathrm{a}$ & $\mathrm{b}$ & $\mathrm{a}$ & $\{\mathrm{a}, \mathrm{b}\}$ \\
$\mathrm{c}$ & $\mathrm{a}$ & $\mathrm{a}$ & $\mathrm{c}$ & $\mathrm{c}$ \\
$\mathrm{d}$ & $\mathrm{a}$ & $\{\mathrm{a}, \mathrm{b}\}$ & $\mathrm{c}$ & $\mathrm{d}$
\end{tabular}

\begin{tabular}{c|cccc}
$\oplus$ & $\mathrm{a}$ & $\mathrm{b}$ & $\mathrm{c}$ & $\mathrm{d}$ \\
\hline $\mathrm{a}$ & $\mathrm{a}$ & $\mathrm{b}$ & $\{\mathrm{c}, \mathrm{d}\}$ & $\mathrm{d}$ \\
$\mathrm{b}$ & $\mathrm{b}$ & $\mathrm{b}$ & $\mathrm{d}$ & $\mathrm{d}$ \\
$\mathrm{c}$ & $\{\mathrm{c}, \mathrm{d}\}$ & $\mathrm{d}$ & $\{\mathrm{c}, \mathrm{d}\}$ & $\mathrm{d}$ \\
$\mathrm{d}$ & $\mathrm{d}$ & $\mathrm{d}$ & $\mathrm{d}$ & $\mathrm{d}$
\end{tabular}

It is easy to check that $(L, \otimes, \oplus)$ is a hyperlattice. Consider the subsets $S_{1}=\{a, d\}$, $S_{2}=\{c, d\}$. Then $S_{1}$ and $S_{2}$ are subhyperlattices of $L$. If we get $S_{3}=\{a, c\}$, then $S_{3}$ is not a subhyperlattice of $L$. Because it isn't closed under the hyperoperation $\oplus$ on $L$.

2.4. Definition. [14] Let $L_{1}=\left(L_{1}, \otimes_{1}, \oplus_{1}\right)$ and $L_{2}=\left(L_{2}, \otimes_{2}, \oplus_{2}\right)$ be two hyperlattices. A map $\varphi: L_{1} \rightarrow L_{2}$ is called a

(i) weak hyperlattice homomorphism if $\varphi\left(a \otimes_{1} b\right) \subseteq \varphi(a) \otimes_{2} \varphi(b)$ and $\varphi\left(a \oplus_{1} b\right) \subseteq$ $\varphi(a) \oplus_{2} \varphi(b)$ for all $a, b \in L_{1}$,

(ii) strong hyperlattice homomorphism if $\varphi\left(a \otimes_{1} b\right)=\varphi(a) \otimes_{2} \varphi(b)$ and $\varphi\left(a \oplus_{1} b\right)=$ $\varphi(a) \oplus_{2} \varphi(b)$ for all $a, b \in L_{1}$.

If such a homomorphism $\varphi$ is surjective, injective or bijective, then $\varphi$ is called an epimorphism, a monomorphism or an isomorphism from the hyperlattice $\left(L_{1}, \otimes_{1}, \oplus_{1}\right)$ to the hyperlattice $\left(L_{2}, \otimes_{2}, \oplus_{2}\right)$, respectively.

2.5. Definition. Let $L=(L, \otimes, \oplus)$ be a hyperlatice and $A \in P^{*}(L)$. Then $A$ is called a hyperideal of $L$ if and only if $a \otimes x \in P^{*}(A), a \oplus x \in P^{*}(A)$ for all $a \in A, x \in L$.

Let $(L, \otimes, \oplus)$ be a hyperlattice. An equivalence relation $\theta$ is a reflexive, symmetric, and transitive binary relation on $L$. If $\theta$ is an equivalence relation on $L$, then the equivalence class of $a \in L$ is the set $\{y \in L \mid(a, y) \in \theta\}$. We write it as $[a]_{\theta}$.

Let $\theta$ be an equivalence relation on $L$. For any $A, B \in P^{*}(L)$, we write that $A \bar{\theta} B$ if the following two conditions are hold:

(1) $\forall a \in A, \exists b \in B$ such that $a \theta b$; (2) $\forall x \in B, \exists y \in A$ such that $x \theta y$. 
We denote $A \overline{\bar{\theta}} B$ if for all $a \in A, b \in B$ we have $a \theta b$.

2.6. Definition. [32] An equivalence relation $\theta$ on a hyperlattice $L=(L, \otimes, \oplus)$ is called a regular (strongly regular) hypercongruence relation if for every $x \in L,(a, b) \in \theta$ implies

$(a \otimes x) \bar{\theta}(b \otimes x) \quad$ and $\quad(a \oplus x) \bar{\theta}(b \oplus x)((a \otimes x) \overline{\bar{\theta}}(b \otimes y) \quad$ and $\quad(a \oplus x) \overline{\bar{\theta}}(b \oplus y))$.

Clearly, any strongly regular hypercongruence relation is a regular hypercongruence relation.

2.7. Example. Let $L=\{a, b, c, d\}$ and let the hyperoperations ${ }^{\prime \prime} \otimes$ " and $" \oplus$ " on $L$ be defined as follows:

\begin{tabular}{c|cccc}
$\otimes$ & $\mathrm{a}$ & $\mathrm{b}$ & $\mathrm{c}$ & $\mathrm{d}$ \\
\hline $\mathrm{a}$ & $\mathrm{a}$ & $\mathrm{a}$ & $\mathrm{a}$ & $\mathrm{a}$ \\
$\mathrm{b}$ & $\mathrm{a}$ & $\{\mathrm{a}, \mathrm{b}\}$ & $\mathrm{a}$ & $\{\mathrm{a}, \mathrm{b}\}$ \\
$\mathrm{c}$ & $\mathrm{a}$ & $\mathrm{a}$ & $\mathrm{c}$ & $\mathrm{c}$ \\
$\mathrm{d}$ & $\mathrm{a}$ & $\{\mathrm{a}, \mathrm{b}\}$ & $\mathrm{c}$ & $\{\mathrm{c}, \mathrm{d}\}$
\end{tabular}

\begin{tabular}{c|cccc}
$\oplus$ & $\mathrm{a}$ & $\mathrm{b}$ & $\mathrm{c}$ & $\mathrm{d}$ \\
\hline $\mathrm{a}$ & $\{\mathrm{a}, \mathrm{b}\}$ & $\mathrm{b}$ & $\{\mathrm{c}, \mathrm{d}\}$ & $\mathrm{d}$ \\
$\mathrm{b}$ & $\mathrm{b}$ & $\mathrm{b}$ & $\mathrm{d}$ & $\mathrm{d}$ \\
$\mathrm{c}$ & $\{\mathrm{c}, \mathrm{d}\}$ & $\mathrm{d}$ & $\{\mathrm{c}, \mathrm{d}\}$ & $\mathrm{d}$ \\
$\mathrm{d}$ & $\mathrm{d}$ & $\mathrm{d}$ & $\mathrm{d}$ & $\mathrm{d}$
\end{tabular}

Then $(L, \otimes, \oplus)$ is a hyperlattice [14]. Let $\theta$ be a hypercongruence relation on the hyperlattice $L$ with the following equivalence classes: $[a]_{\theta}=[b]_{\theta}=\{a, b\},[c]_{\theta}=[d]_{\theta}=\{c, d\}$. Then $\theta$ is a strongly regular hypercongruence relation on $L$.

2.8. Definition. Let $L=(L, \otimes, \oplus)$ be a hyperlattice and $\theta$ be a regular hypercongruence relation on $L$. Then $\theta$ is called a complete hypercongruence relation if $[a \otimes b]_{\theta}=\left\{x \otimes y \mid x \in[a]_{\theta}, y \in[b]_{\theta}\right\}$, and $[a \oplus b]_{\theta}=\left\{x \oplus y \mid x \in[a]_{\theta}, y \in[b]_{\theta}\right\}$ for all $a, b \in L$.

2.9. Example. Let $L=\{0, a, b, c, 1\}$ be a lattice $(L, \wedge, \vee)$, where the partial order relation on $L$ is defined as shown in Figure 1. For all $x, y \in L, x \otimes y=\{x \wedge y\}$, $x \oplus y=\{x \vee y\}$, then $L=(L, \otimes, \oplus)$ is a hyperlattice.

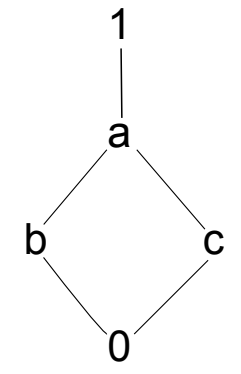

Figure 1. The lattice in Example 2.9.

(i) Let $\theta$ be a regular hypercongruence relation on the hyperlattice $L$ with the following equivalence classes: $[1]_{\theta}=1,[a]_{\theta}=[c]_{\theta}=\{a, c\},[b]_{\theta}=[0]_{\theta}=\{b, 0\}$. Then $\theta$ is a complete hypercongruence relation.

(ii) Let $\theta$ be a regular hypercongruence relation on the hyperlattice $L$ with the following equivalence classes: $[1]_{\theta}=[a]_{\theta}=\{1, a\},[c]_{\theta}=\{c\},[b]_{\theta}=\{b\},[0]_{\theta}=$ $\{0\} . \theta$ is not complete because $[c \oplus b]_{\theta}=\{1, a\},[c]_{\theta} \oplus[b]_{\theta}=\{a\}$ and $[c \oplus b]_{\theta} \neq$ $[c]_{\theta} \oplus[b]_{\theta}$. 
2.10. Lemma. Let $L=(L, \otimes, \oplus)$ be a hyperlattice and $\theta$ be a regular hypercongruence relation on $L$. Then for all $a, b, c, d \in L$,

(i) If $(a, b) \in \theta$ and $(c, d) \in \theta$, then $(a \otimes c) \bar{\theta}(b \otimes d)$ and $(a \oplus c) \bar{\theta}(b \oplus d)$,

(ii) $\left\{x \otimes y \mid x \in[a]_{\theta}, y \in[b]_{\theta}\right\} \subseteq[a \otimes b]_{\theta}$,

(iii) $\left\{x \oplus y \mid x \in[a]_{\theta}, y \in[b]_{\theta}\right\} \subseteq[a \oplus b]_{\theta}$.

\section{Rough subsets of a hyperlattice in the generalized approxima- tion space}

In this section, according to the notion of generalized approximation space presented in $[9,35,36]$, we present some basic concepts about the generalized approximation space $(U, W, T)$ and the associated lower and upper approximation operators. Let $U$ and $W$ be two non-empty universes. Let $T$ be a set-valued mapping given by $T: U \rightarrow P(W)$. Then the triple $(U, W, T)$ is referred to as a generalized approximation space. Any setvalued function from $U$ to $P(W)$ defines a binary relation from $U$ to $W$ by setting $\rho_{T}=\{(x, y) \mid y \in T(x)\}$. Obviously, if $\rho$ is an arbitrary relation from $U$ to $W$, then it can be defined as a set-valued mapping $T_{\rho}: U \rightarrow P(W)$ by $T_{\rho}(x)=\{y \in W \mid(x, y) \in \rho\}$, where $x \in U$. For any set $X \subseteq W$, a pair of lower and upper approximations $\underline{T}(X)$ and $\bar{T}(X)$, are defined by

$\underline{T}(X)=\{x \in U \mid T(x) \subseteq X\}$ and $\bar{T}(X)=\{x \in U \mid T(x) \cap X \neq \emptyset\}$. The pair $(\underline{T}(X), \bar{T}(X))$ is referred to as a generalized rough set and $\underline{T}$ and $\bar{T}$ are referred to as lower and upper generalized approximation operators, respectively.

3.1. Definition. Let $L_{1}=\left(L_{1}, \otimes_{1}, \oplus_{1}\right)$ and $L_{2}=\left(L_{2}, \otimes_{2}, \oplus_{2}\right)$ be two hyperlattices. A mapping $T: L_{1} \rightarrow P\left(L_{2}\right)$ is called a set-valued homomorphism if for all $a, b \in L_{1}$,

(i) $T(a) \otimes_{2} T(b) \subseteq T\left(a \otimes_{1} b\right)$,

(ii) $T(a) \oplus_{2} T(b) \subseteq T\left(a \oplus_{1} b\right)$.

3.2. Definition. Let $L_{1}=\left(L_{1}, \otimes_{1}, \oplus_{1}\right)$ and $L_{2}=\left(L_{2}, \otimes_{2}, \oplus_{2}\right)$ be two hyperlattices. A mapping $T: L_{1} \rightarrow P\left(L_{2}\right)$ is called a strong set-valued homomorphism if for all $a, b \in L_{1}$,

(i) $T(a) \otimes_{2} T(b)=T\left(a \otimes_{1} b\right)$,

(ii) $T(a) \oplus_{2} T(b)=T\left(a \oplus_{1} b\right)$.

3.3. Example. Let $L_{1}=\left(L_{1}, \otimes_{1}, \oplus_{1}\right)$ and $L_{2}=\left(L_{2}, \otimes_{2}, \oplus_{2}\right)$ be two hyperlattices.

(i) The set-valued map $T: L_{1} \rightarrow P\left(L_{2}\right)$ defined by $T(a)=L_{2}$ is a set-valued homomorphism.

(ii) If $\theta$ is a regular hypercongruence relation on a hyperlattice $L_{1}$ then $T_{\theta}: L_{1} \rightarrow$ $P\left(L_{1}\right)$ defined by $T_{\theta}(a)=[a]_{\theta}$ is a set-valued homomorphism. If $\theta$ is a complete regular hypercongruence then $T_{\theta}$ is a strong set-valued homomorphism.

(iii) If $\varphi: L_{1} \rightarrow L_{2}$ is a strong hyperlattice homomorphism, then the set-valued map $T: L_{1} \rightarrow P\left(L_{2}\right)$ defined by $T(a)=\{\varphi(a)\}$ is a strong set-valued homomorphism.

Note that Example 3.3. (ii) indicates that every regular hyper congruence relations may be considered as a set-valued homomorphism. On the other hand, hypercongruence relations are important in hyperalgebraic systems. So set-valued homomorphisms are interesting for pure algebraic systems.

3.4. Proposition. Let $L_{1}=\left(L_{1}, \otimes_{1}, \oplus_{1}\right)$ and $L_{2}=\left(L_{2}, \otimes_{2}, \oplus_{2}\right)$ be two hyperlattices and $T: L_{1} \rightarrow P\left(L_{2}\right)$ be a set valued homomorphism. If $X, Y \in P^{*}\left(L_{2}\right)$, then 
(i) $\bar{T}(X) \otimes_{1} \bar{T}(Y) \subseteq \bar{T}\left(X \otimes_{2} Y\right)$,

(ii) $\bar{T}(X) \oplus_{1} \bar{T}(Y) \subseteq \bar{T}\left(X \oplus_{2} Y\right)$.

Proof. (i) Assume that $x \in \bar{T}(X) \otimes_{1} \bar{T}(Y)$. Then $x \in x_{1} \otimes_{1} x_{2}$ with $x_{1} \in \bar{T}(X), x_{2} \in \bar{T}(Y)$. Hence $T\left(x_{1}\right) \cap X \neq \emptyset$ and $T\left(x_{2}\right) \cap Y \neq \emptyset$. Then there exist $a \in T\left(x_{1}\right) \cap X$ and $b \in T\left(x_{2}\right) \cap Y$ such that $a \in T\left(x_{1}\right), b \in T\left(x_{2}\right)$ and $a \in X, b \in Y$. Therefore $a \otimes_{2} b \subseteq X \otimes_{2} Y$. Since $T$ is a set-valued homomorphism, we have $a \otimes_{2} b \subseteq T\left(x_{1}\right) \otimes_{2} T\left(x_{2}\right) \subseteq T\left(x_{1} \otimes_{1} x_{2}\right)$. Hence $T\left(x_{1} \otimes_{1} x_{2}\right) \cap\left(X \otimes_{2} Y\right) \neq \emptyset$ which implies that $x \in \bar{T}\left(X \otimes_{2} Y\right)$. So $\bar{T}(X) \otimes_{1} \bar{T}(Y) \subseteq$ $\bar{T}\left(X \otimes_{2} Y\right)$.

(ii) The proof is similar to (i).

3.5. Corollary. Let $\theta$ be a regular hypercongruence relation on a hyperlattice $L$ and $X, Y \in P^{*}(L)$. Then

(i) $\overline{T_{\theta}}(X) \otimes \overline{T_{\theta}}(Y) \subseteq \overline{T_{\theta}}(X \otimes Y)$,

(ii) $\overline{T_{\theta}}(X) \oplus \overline{T_{\theta}}(Y) \subseteq \overline{T_{\theta}}(X \oplus Y)$.

The following example shows that the inclusion symbol " $\subseteq$ " in Propositions 3.4. may not be replaced by the equal sign.

3.6. Example. Consider the hyperlattice defined in Example 2.3. Let $T: L \rightarrow P(L)$ be a set-valued map defined as $T(x)=\{a\}$. Then it is easy to see that $T$ is a set-valued homomorphism. If $X=\{b\}$ and $Y=\{d\}$, then $\bar{T}(X) \otimes \bar{T}(Y)=\emptyset, \bar{T}(X \otimes Y)=L$. Thus $\bar{T}(X) \otimes \bar{T}(Y) \neq \bar{T}(X \otimes Y)$. Further, if $T: L \rightarrow P(L)$ is a set-valued map defined as $T(x)=\{d\}$, then $T$ is a set-valued homomorphism. If $X=Y=\{c\}$, then $\bar{T}(X) \oplus \bar{T}(Y)=\emptyset$, then $\bar{T}(X \oplus Y)=L$. Thus $\bar{T}(X) \oplus \bar{T}(Y) \neq \bar{T}(X \oplus Y)$.

3.7. Proposition. Let $L_{1}=\left(L_{1}, \otimes_{1}, \oplus_{1}\right), L_{2}=\left(L_{2}, \otimes_{2}, \oplus_{2}\right)$ be two hyperlattices and $T: L_{1} \rightarrow P\left(L_{2}\right)$ be a strong set valued homomorphism. If $X, Y \in P^{*}\left(L_{2}\right)$, then

(i) $\underline{T}(X) \otimes_{1} \underline{T}(Y) \subseteq \underline{T}\left(X \otimes_{2} Y\right)$,

(ii) $\underline{T}(X) \oplus_{1} \underline{T}(Y) \subseteq \underline{T}\left(X \oplus_{2} Y\right)$.

Proof. (i) Assume that $z \in \underline{T}(X) \otimes_{1} \underline{T}(Y)$. Then $z \in x \otimes_{1} y$ with $x \in \underline{T}(X), y \in \underline{T}(Y)$. Hence $T(x) \subseteq X$ and $T(y) \subseteq Y$. Since $T$ is a strong set-valued homomorphism, we have $T(x) \otimes_{2} T(y)=T\left(x \otimes_{1} x\right) \subseteq A \otimes_{2} B$. Hence $z \in x \otimes_{2} y \in \underline{T}\left(X \otimes_{2} Y\right)$, that is $\underline{T}(X) \otimes_{1} \underline{T}(Y) \subseteq \underline{T}\left(X \otimes_{2} Y\right)$.

(ii) The proof is similar to (i).

3.8. Corollary. Let $\theta$ be a regular hypercongruence relation on a hyperlattice $L$ and $X, Y \in P^{*}(L)$. Then

(i) $T_{\theta}(X) \otimes T_{\theta}(Y) \subseteq T_{\theta}(X \otimes Y)$,

(ii) $\overline{T_{\theta}}(X) \oplus \overline{T_{\theta}}(Y) \subseteq \underline{T_{\theta}}(X \oplus Y)$.

The following example shows that the containment in the above proposition is proper.

3.9. Example. Consider the hyperlattice defined in Example 2.3. Let $T: L \rightarrow P(L)$ be a set-valued map defined as $T(x)=\{a\}$. Then it is easy to see that $T$ is a setvalued homomorphism. If $X=\{d\}, Y=\{b\}$, then $\underline{T}(X) \otimes \underline{T}(Y)=\emptyset, \underline{T}(X \otimes Y)=L$. 
Thus $\underline{T}(X) \otimes \underline{T}(Y) \neq \underline{T}(X \otimes Y)$. Further, if $T: L \rightarrow P(L)$ is a set-valued map defined as $T(x)=\{d\}$, then $T$ is a set-valued homomorphism. If $X=Y=\{c\}$, then $\underline{T}(X) \oplus \underline{T}(Y)=\emptyset, \underline{T}(X \oplus Y)=L$. Thus $\underline{T}(X) \oplus \underline{T}(Y) \neq \underline{T}(X \oplus Y)$.

3.10. Proposition. Let $T: L_{1} \rightarrow P\left(L_{2}\right)$ be a (strong) set-valued homomorphism and $f: L_{3} \rightarrow L_{1}$ be a weak (strong) hyperlattice homomorphism. Then $T \circ f$ is a (strong) set-valued homomorphism from $L_{3} \rightarrow P\left(L_{2}\right)$ such that $\overline{T \circ f}(X)=f^{-1}(\bar{T}(X))$ and $\underline{T \circ f}(X)=f^{-1}(\underline{T}(X))$, for all $X \in P\left(L_{2}\right)$.

Proof. The proof is straightforward.

3.11. Proposition. Let $T: L_{1} \rightarrow P\left(L_{2}\right)$ be a (strong) set-valued homomorphism and $f: L_{2} \rightarrow L_{3}$ be a weak (strong) hyperlattice homomorphism. Then $T_{f}$ is a (strong) set-valued homomorphism from $L_{1} \rightarrow P\left(L_{3}\right)$ defined by $T_{f}(r)=f(T(r))$ such that $\underline{T_{f}}(X)=\underline{T}\left(f^{-1}(X)\right)$ and $\overline{T_{f}}(X)=\bar{T}\left(f^{-1}(X)\right)$, for all $X \in P\left(L_{3}\right)$.

Proof. The proof is straightforward.

3.12. Definition. Let $L_{1}=\left(L_{1}, \otimes_{1}, \oplus_{1}\right), L_{2}=\left(L_{2}, \otimes_{2}, \oplus_{2}\right)$ be two hyperlattices and let $T: L_{1} \rightarrow P\left(L_{2}\right)$ be a set-valued mapping. If $\bar{T}(X)$ and $\underline{T}(X)$ are subhyperlattices (resp. hyperideals) of $L_{1}$, then $(\bar{T}(X), \underline{T}(X))$ is called a generalized rough subhyperlattice (resp. hyperideal).

3.13. Example. Let $L=(L, \otimes, \oplus)$ be a hyperlattice defined in Example 2.3. Let $T: L \rightarrow P(L)$ be a set-valued map defined as $T(x)=\{b\}$ and $X=\{a, b\}$. Then $\bar{T}(X)$ and $\underline{T}(X)$ are subhyperlattices (resp. hyperideals) of $L$. Hence $(\bar{T}(X), \underline{T}(X))$ is a generalized rough subhyperlattice (resp. hyperideal).

3.14. Theorem. Let $L_{1}=\left(L_{1}, \otimes_{1}, \oplus_{1}\right), L_{2}=\left(L_{2}, \otimes_{2}, \oplus_{2}\right)$ be two hyperlattices and $X \in P^{*}\left(L_{2}\right)$.

(i) If $T: L_{1} \rightarrow P\left(L_{2}\right)$ is a set-valued homomorphism and $X$ is a subhyperlattice of $L_{2}$, then $\bar{T}(X)$ is a subhyperlattice of $L_{1}$.

(ii) If $T: L_{1} \rightarrow P\left(L_{2}\right)$ is a strong set-valued homomorphism and $X$ is a subhyperlattice of $L_{2}$, then $\underline{T}(X)$ is, if it is non-empty, a subhyperlattice of $L_{1}$.

(iii) If $T: L_{1} \rightarrow P^{*}\left(L_{2}\right)$ is a set-valued homomorphism and $X$ is a hyperideal of $L_{2}$, then $\bar{T}(X)$ is a hyperideal of $L_{1}$.

(iv) If $T: L_{1} \rightarrow P^{*}\left(L_{2}\right)$ is a strong set-valued homomorphism and $X$ is a hyperideal of $L_{2}$, then $\underline{T}(X)$ is, if it is non-empty, a hyperideal of $L_{1}$.

Proof. (i) Suppose that $x, y \in \bar{T}(X)$. Then $T(x) \cap X \neq \emptyset$ and $T(y) \cap X \neq \emptyset$. Hence there exist $a \in T(x) \cap X$ and $b \in T(y) \cap X$. Thus $a \otimes_{2} b \subseteq T(x) \otimes_{2} T(y) \subseteq T\left(x \otimes_{1} y\right)$ and $a \oplus_{2} b \subseteq T(x) \oplus_{2} T(y) \subseteq T\left(x \oplus_{1} y\right)$. Since $X$ is a subhyperlattice of $L_{2}$, we have $a \otimes_{2} b \subseteq X$ and $a \oplus_{2} b \subseteq X$. So $T\left(x \otimes_{1} y\right) \cap X \neq \emptyset$ and $T\left(x \oplus_{1} y\right) \cap X \neq \emptyset$. Therefore $x \otimes_{1} y, x \oplus_{1} y \in \bar{T}(X)$. Consequently, $\bar{T}(X)$ is a subhyperlattice of $L_{1}$.

(ii) Suppose that $x, y \in \underline{T}(X)$. Then $T(x) \subseteq X$ and $T(y) \subseteq X$. Since $X$ is a subhyperlattice of $L_{2}$ and $T$ is a strong set-valued homomorphism, we have $T\left(x \otimes_{1} y\right)=$ 
$T(x) \otimes_{2} T(y) \subseteq X \otimes_{2} X \subseteq X$ and $T\left(x \oplus_{1} y\right)=T(x) \oplus_{2} T(y) \subseteq X \oplus_{2} X \subseteq X$. Thus $x \otimes_{1} y, x \oplus_{1} y \in \underline{T}(X)$. Therefore $\underline{T}(X)$ is a subhyperlattice of $L_{1}$.

(iii) By (i) $\bar{T}(X)$ is a subhyperlattice of $L_{1}$. Let $b \in L_{1}$. Since $T(b) \neq \emptyset$, there exist some $z \in L_{2}$ such that $z \in T(b)$. Let $x \in \bar{T}(X)$. Then $T(x) \cap X \neq \emptyset$ which implies that there exists $a \in T(x) \cap X$, that is $a \in T(x), a \in X$. Since $X$ is a hyperideal of $L_{2}$ and $T$ is a strong set-valued homomorphism, we have $a \otimes_{2} z, a \oplus_{2} z \subseteq X$ and $a \otimes_{2} z \subseteq T(x) \otimes_{2} T(b)=T\left(x \otimes_{1} b\right), a \oplus_{2} z \subseteq T(x) \oplus_{2} T(b)=T\left(x \oplus_{1} b\right)$ which implies that $T\left(x \otimes_{1} b\right) \cap X \neq \emptyset$ and $T\left(x \oplus_{1} b\right) \cap X \neq \emptyset$. Thus $x \otimes_{1} b, x \oplus_{1} b \in \bar{T}(X)$. Therefore $\bar{T}(X)$ is a hyperideal of $L_{1}$.

(iv) Similarly, $\underline{T}(X)$ is a hyperideal of $L_{1}$.

The following example shows that the converse of the above theorem does not hold in general.

3.15. Example. Consider the hyperlattice defined Example 2.3. Let $T: L \rightarrow P(L)$ be a set-valued map defined as $T(x)=\{d\}$. Then it is easy to see that $T$ is a set-valued homomorphism. If $X=\{b, d\}$, then $X$ is not a subhyperlattice (hyperideal) of $L$. But $\bar{T}(X)=L$ is a subhyperlattice (hyperideal) of $L$.

3.16. Corollary. Let $\theta$ be a regular hypercongruence relation on a hyperlattice $L=$ $(L, \otimes, \oplus)$.

(i) If $X$ is a hyperlattice of $L$, then $\overline{T_{\theta}}(X)$ is a subhyperlattice of $L$.

(ii) If $\theta$ is a complete regular hypercongruence relation and $X$ is a subhyperlattice of $L$, then $\underline{T}_{\theta}(X)$ is, if it is non-empty, a subhyperlattice of $L$.

(iii) If $X$ is a hyperideal of $L$, then $\overline{T_{\theta}}(X)$ is a hyperideal of $L$.

(iv) If $\theta$ is a complete regular hypercongruence relation and $X$ is a hyperideal of $L$, then $T_{\theta}(X)$ is, if it is non-empty, a hyperideal of $L$.

Now we give a counterexample which shows that the condition that $\theta$ is a complete regular hypercongruence relation in Corollary 3.16. is necessary.

3.17. Example. Consider the hyperlattice $L$ and the congruence relation on $L$ defined in Example 2.9.(ii). If $X=\{a, b, c, 0\}$, then $X$ is a subhyperlattice of $L$. But $\underline{T_{\theta}}(X)=\{b, c, 0\}$ is not a subhyperlattice of $L$.

\section{Generalized lower and upper approximation operators with re- spect to a hyperideal of a hyperlattice}

4.1. Definition. Let $L_{1}=\left(L_{1}, \otimes_{1}, \oplus_{1}\right), L_{2}=\left(L_{2}, \otimes_{2}, \oplus_{2}\right)$ be two hyperlattices, $A$ be a hyperideal of $L_{2}$ and $T: L_{1} \rightarrow P\left(L_{2}\right)$ be a set-valued mapping. Then we define $T_{A}: L_{1} \rightarrow P\left(L_{2}\right)$ as $T_{A}(a)=T(a) \otimes_{2} A$ for all $a \in L_{1}$. Then $T_{A}$ is called the set-valued mapping with respect to a hyperideal $A$.

4.2. Definition. Let $\left(L_{1}, L_{2}, T_{A}\right)$ be a generalized approximation space with respect to a hyperideal $A$ and $X$ be a non-empty subset of $L_{2}$. Then the sets $\underline{T_{A}}(X)=\left\{a \in L_{1} \mid T_{A}(a) \subseteq X\right\}$ and $\overline{T_{A}}(X)=\left\{a \in L_{1} \mid T_{A}(a) \cap X \neq \emptyset\right\}$ 
are called generalized lower and upper approximations of $X$ with respect to the hyperideal $A$, respectively.

4.3. Lemma. Let $L_{1}=\left(L_{1}, \otimes_{1}, \oplus_{1}\right), L_{2}=\left(L_{2}, \otimes_{2}, \oplus_{2}\right)$ be two hyperlattices and $A, B$ be hyperideals of $L_{2}$. Let $X$ be a subset of $L_{2}$ such that $A \subseteq B$. Then

(i) $\overline{T_{A}}(X) \subseteq \overline{T_{B}}(X)$,

(ii) $\underline{T_{B}}(X) \subseteq \underline{T_{A}}(X)$.

Proof. (i) Suppose that $x \in \overline{T_{A}}(X)$. Then $\left(T(x) \otimes_{2} A\right) \cap X \neq \emptyset$. So there exist $a \in$ $\left(T(x) \otimes_{2} A\right) \cap X$ such that $a \in\left(T(x) \otimes_{2} A\right)$ and $a \in X$. Hence there exist $y \in T(x), z \in A$ such that $a=y \otimes_{2} z$. Since $A \subseteq B$, we have $z \in B$. Thus $a=y \otimes_{2} z \subseteq T(x) \otimes_{2} B$ and $a \in X$. So $\left(T(x) \otimes_{2} B\right) \cap X \neq \emptyset$. As a consequent, we obtain $\overline{T_{A}}(X) \subseteq \overline{T_{B}}(X)$.

(ii) The proof is similar to (i).

The following corollary follows from Lemma 4.3 .

4.4. Corollary. Let $L_{1}=\left(L_{1}, \otimes_{1}, \oplus_{1}\right), L_{2}=\left(L_{2}, \otimes_{2}, \oplus_{2}\right)$ be two hyperlattices and $A, B$ be hyperideals of $L_{2}$. Let $X$ be a subset of $L_{2}$ such that $A \subseteq B$. Then

(i) $\overline{T_{A \cap B}}(X) \subseteq \overline{T_{A}}(X) \cap \overline{T_{B}}(X)$,

(ii) $\underline{T_{A}}(X) \cap \underline{T_{B}}(X) \subseteq \underline{T_{A \cap B}}(X)$.

4.5. Proposition. Let $\left(L_{1}, L_{2}, T_{A}\right)$ be a generalized approximation with respect to a hyperideal $A$ and $X, Y$ be a non-empty subsets of $L_{2}$.

(i) If $T: L_{1} \rightarrow P\left(L_{2}\right)$ is a set-valued homomorphism, then $\overline{T_{A}}(X) \otimes_{1} \overline{T_{A}}(Y) \subseteq$ $\overline{T_{A}}\left(X \otimes_{2} Y\right)$.

(ii) If $T: L_{1} \rightarrow P\left(L_{2}\right)$ is a strong set-valued homomorphism, then $\underline{T_{A}}(X) \otimes_{1} \underline{T_{A}}(Y) \subseteq$ $\underline{T_{A}}\left(X \otimes_{2} Y\right)$.

Proof. (i) Suppose that $z \in \overline{T_{A}}(X) \otimes_{1} \overline{T_{A}}(Y)$. Then there exist $x \in \overline{T_{A}}(X), y \in \overline{T_{A}}(Y)$ such that $z \in x \otimes_{1} y$. Since $x \in \overline{T_{A}}(X) y \in \overline{T_{A}}(Y)$ there exist $a \in T(x) \otimes_{2} A, b \in T(y) \otimes_{2} A$ such that $a \in T(x), b \in T(y), a \in X, b \in Y$. Since $T$ is a set-valued homomorphism, we have $a \otimes_{2} b \subseteq T(x) \otimes_{2} T(y) \otimes_{2} A \subseteq T\left(x \otimes_{1} y\right) \otimes_{2} A$ and $a \otimes_{2} b \subseteq X \otimes_{2} Y$. Hence $a \otimes_{2} b \subseteq T\left(x \otimes_{1} y\right) \otimes_{2} A \cap\left(X \otimes_{2} Y\right)$. So $z \in x \otimes_{1} y \subseteq \overline{T_{A}}\left(X \otimes_{2} Y\right)$.Therefore, we obtain $\overline{T_{A}}(X) \otimes_{1} \overline{T_{A}}(Y) \subseteq \overline{T_{A}}\left(X \otimes_{2} Y\right)$.

(ii) The proof is similar to (i).

4.6. Proposition. Let $L_{1}=\left(L_{1}, \otimes_{1}, \oplus_{1}\right), L_{2}=\left(L_{2}, \otimes_{2}, \oplus_{2}\right)$ be two hyperlattices, $A, B$ be hyperideals of $L_{2}$ and $X$ be a subhyperlatice of $L_{2}$.

(i) If $T: L_{1} \rightarrow P\left(L_{2}\right)$ is a set-valued homomorphism, then $\overline{T_{A}}(X) \otimes_{1} \overline{T_{B}}(X) \subseteq$ $\overline{T_{A \otimes_{2} B}}(X)$.

(ii) If $T: L_{1} \rightarrow P\left(L_{2}\right)$ is a strong set-valued homomorphism, then $\underline{T_{A}}(X) \otimes_{1}$ $\underline{T_{B}}(X)=\underline{T_{A \otimes_{2} B}}(X)$.

Proof. The proof is straightforward.

4.7. Theorem. Let $\left(L_{1}, L_{2}, T_{A}\right)$ be a generalized approximation space with respect to a hyperideal $A$ and $X$ be a non-empty subset of $L_{2}$.

(i) If $T: L_{1} \rightarrow P\left(L_{2}\right)$ is a set-valued homomorphism and $X$ is a subhyperlattice of $L_{2}$, then $\overline{T_{A}}(X)$ is a subhyperlattice of $L_{1}$. 
(ii) If $T: L_{1} \rightarrow P\left(L_{2}\right)$ is a strong set-valued homomorphism and $X$ is a subhyperlattice of $L_{2}$, then $T_{A}(X)$ is, if it is non-empty, a subhyperlattice of $L_{1}$.

(iii) If $T: L_{1} \rightarrow P^{*}\left(L_{2}\right)$ is a set-valued homomorphism and $X$ is a hyperideal of $L_{2}$, then $\overline{T_{A}}(X)$ is a hyperideal of $L_{1}$.

(iv) If $T: L_{1} \rightarrow P^{*}\left(L_{2}\right)$ be a strong set-valued homomorphism and $X$ is a hyperideal of $L_{2}$, then $\underline{T_{A}}(X)$ is, if it is non-empty, a hyperideal of $L_{1}$.

Proof. (i) Suppose that $x, y \in \overline{T_{A}}(X)$. Then, $\left(T(x) \otimes_{2} A\right) \cap X \neq \emptyset$ and $\left(T(y) \otimes_{2} A\right) \cap X \neq$ $\emptyset$. Hence there exist $a \in\left(T(x) \otimes_{2} A\right) \cap X$ and $b \in\left(T(y) \otimes_{2} A\right) \cap X$. Since $X$ is a subhyperlattice of $L_{2}$, we have $a \otimes_{2} b \subseteq X$ and $a \oplus_{2} b \subseteq X$. On the other hand, $a \otimes_{2} b \subseteq\left(T(x) \otimes_{2} A\right) \otimes_{2}\left(T(y) \otimes_{2} A\right) \subseteq T(x) \otimes_{2} T(y) \otimes_{2} A \subseteq T\left(x \otimes_{1} y\right) \otimes_{2} A$ and $a \oplus_{2} b \subseteq\left(T(x) \otimes_{2} A\right) \oplus_{2}\left(T(y) \otimes_{2} A\right) \subseteq T(x) \oplus_{2} T(y) \otimes_{2} A \subseteq T\left(x \oplus_{1} y\right) \otimes_{2} A$. So $T\left(x \otimes_{1} y\right) \otimes_{2} A \cap X \neq \emptyset$ and $T\left(x \oplus_{1} y\right) \otimes_{2} A \cap X \neq \emptyset$. Thus $x \otimes_{1} y, x \oplus_{1} y \in \overline{T_{A}}(X)$. Therefore, $\overline{T_{A}}(X)$ is a subhyperlattice of $L_{1}$.

(ii) Similarly, $T_{A}(X)$ is a subhyperlattice of $L_{1}$.

(iii) Using (i), $\overline{T_{A}}(X)$ is a subhyperlattice of $L_{1}$. Let $x \in \overline{T_{A}}(X)$ and $c \in L_{1}$. Then $\left(T(x) \otimes_{2} A\right) \cap X \neq \emptyset$. So there exist $a \in\left(T(x) \otimes_{2} A\right) \cap X$. Since $\overline{T_{A}}(X)$ is non-empty set, we can choose $z \in T(c)$. Since $X$ is a hyperideal of $L_{2}$, we have $a \otimes_{2} z, a \oplus_{2} z \subseteq X$. On the other hand, $a \otimes_{2} z \subseteq\left(T(x) \otimes_{2} A\right) \otimes_{2} T(c) \subseteq T\left(x \otimes_{1} c\right) \otimes_{2} A, a \oplus_{2} z \subseteq\left(T(x) \otimes_{2} A\right) \oplus_{2} T(c) \subseteq$ $T\left(x \oplus_{1} c\right) \otimes_{2} A$. So $\left(T\left(x \otimes_{1} c\right) \otimes_{2} A\right) \cap X \neq \emptyset,\left(T\left(x \oplus_{1} c\right) \otimes_{2} A\right) \cap X \neq \emptyset$ which implies $x \otimes_{1} c, x \oplus_{1} c \in \overline{T_{A}}(X)$. Therefore $\overline{T_{A}}(X)$ is a hyperideal of $L_{1}$.

(iv) The proof is straightforward.

The following example shows that the converse of the above theorem does not hold in general.

4.8. Example. Consider the hyperlattice defined in Example 2.9. Let $T: L \rightarrow P(L)$ be a set-valued map defined as $T(x)=\{d\}$. Then it is easy to see that $T$ is a setvalued homomorphism. If $A=L, X=\{a, b, c\}$, then $A$ is a hyperideal and $X$ is not a subhyperlattice (hyperideal) of $L$. But $\overline{T_{A}}(X)=L$ is a subhyperlattice (hyperideal) of $L$.

\section{Conclusion}

The Pawlak rough sets on algebraic sets such as semigroups, groups, rings, modules and lattices were mainly studied by congruence relations. In this paper, a definition of set-valued homomorphism which was introduced for groups by Davvaz [9], for rings and modules by Yamak et al. [35-36], respectively, is considered as a regular hypercongruence relation for hyperlattices. We obtain some new properties of a set-valued homomorphism to provide opportunity for putting reasonable interpretations on the theory and applications of rough sets and adhering to the set-valued homomorphism and exploring the features of generalized rough approximations on hyperlattices. So, in this paper we propose a definition of set-valued homomorphism and explore the properties of generalized rough approximations on hyperlattices. Some new properties of set-valued homomorphisms which shall be very practical in the theory and applications of rough sets are obtained. Moreover, a new algebraic structure called generalized lower and upper approximations of a set with respect to a hyperideal is presented. 


\section{Acknowledgments}

The authors are highly grateful to referees and Editor-in-Chief, for their valuable comments and suggestions for improving the paper.

\section{References}

[1] Ali, M.I., Shabir, M., Tanveer, S., Roughness in hemirings, Neural Computing and Applications 21, 171-180, 2012.

[2] Anvariyeh, S.M., Mirvakili, S., Davvaz, B., Pawlak's approximations in $\gamma$ semihypergroups, Computers Mathematics with Applications 60, 45-53, 2010.

[3] Ashrafi, A.R., About some join spaces and hyperlattices, Italian Journal of Pure and Applied Mathematics 10, 199-205, 2001.

[4] Biswas, R., Nanda, S., Rough groups and rough subgroups, Bulletin of the Polish Academy of Sciences-Technical Sciences 42, 251-254, 1994.

[5] Corsini, P., Leoreanu-Fotea, V., Applications of Hyperstructure Theory, Kluwer Academic Publishers, Dordrecht, The Netherlands, 2003.

[6] Davvaz, B., Leoreanu-Fotea, V., Hyperring Theory and Applications, International Academic Press, Palm Harbor, Fla, USA, 2007.

[7] Davvaz, B., Mahdavipour, M., Roughness in modules, Information Sciences 176, 3658-3674, 2006.

[8] Davvaz, B., Roughness in rings, Information Sciences 164, 147-163, 2004.

[9] Davvaz, B., A short note on algebraic T-rough sets, Information Sciences 178, 3247-3252, 2008.

[10] Davvaz, B., Mahdavipour, M., Rough approximations in general approximation space and their fundamental properties, International Journal of General Systems 37 (3), 373-386, 2008.

[11] Estaji, A.A., Hooshmandasl, M.R., Davvaz, B., Rough set theory applied to lattice theory, Information Sciences 200, 108-122, 2012.

[12] Han, S.W., Zhao, B., Distributive hyperlattices, J. Northwest Univ. 35, 125-129, 2005 (in Chinese).

[13] Hooshmandasl, M.R., Karimi, A., Almbardara, M., Davvaz, B., Axiomatic systems for rough set-valued homomorphisms of associative rings, International Journal of Approximate Reasoning 54, 297-306, 2013.

[14] Guo, X.Z., Xin, X.L., Hyperlattices, Pure and Applied Mathematics 20, 40-43, 2004.

[15] Marty, F., Sur une generalization de la notion de groupe, in: 8th Congress Math. Scandinaves, Stockholm, 45-49, 1934.

[16] Kazanc1, O., Davvaz, B., On the structure of rough prime (primary) ideals and rough fuzzy prime (primary) ideals in commutative rings, Information Sciences 178, 1343-1354, 2008.

[17] Kazanc1, O., Yamak, S., Davvaz, B., The lower and upper approximations in a quotient hypermodule with respect to fuzzy sets, Information Sciences 178, 2349-2359, 2008.

[18] Konstantinidou, M., Mittas, J., An introduction to the theory of hyperlattices, Mathematica Balkanica 7, 187-193, 1977.

[19] Konstantinidou, M., Distributive and complemented hyperlattices, Prakt. Akad. Athenon 56, 339-360, 1981.

[20] Konstantinidou, M., A representation theorem for P-hyperlattices, Riv. Mat. Pura Appl. 18, 63-69, 1996.

[21] Konstantinidou, M., On P-hyperlattices and their distributivity, Rendiconti del Circolo Matematico di Palermo 42, 391-403, 1993.

[22] Kuroki, N., Rough ideals in semigroups, Information Sciences 100, 139-163, 1997.

[23] Kuroki, N., Wang, P.P., The lower and upper approximations in a fuzzy group, Information Sciences 90, 203-220, 1996.

[24] Leoreanu-Fotea, V., Davvaz, B., Roughness in n-ary hypergroups, Information Sciences 178, 4114-4124, 2008.

[25] Leoreanu-Fotea, V., The lower and upper approximations in a hypergroup, Information Sciences 178, 3605-3615, 2008. 
[26] Pawlak, Z., Rough sets, International Journal of Computer Information Sciences 11, 341$356,1982$.

[27] Pawlak, Z., Rough sets and intelligent data analysis, Information Sciences 147, 1-12, 2002.

[28] Pawlak, Z., Skowron, Z., Rough sets and Boolean reasoning, Information Sciences 177, 41-73, 2007.

[29] Rahnamai-Barghi, A., The prime ideal theorem and semiprime ideals in meet-hyperlattices, Italian Journal of Pure and Applied Mathematics 5, 53-60, 1999.

[30] Rahnamai-Barghi, A., The prime ideal theorem for distributive hyperlattices, Italian Journal of Pure and Applied Mathematics 10, 75-78, 2001.

[31] Rasouli, S., Davvaz, B., Roughness in MV-algebras, Information Sciences 180, 737-747, 2010.

[32] Rasouli, S., Davvaz, B., Lattices derived from hyperlattices, Communications in Algebra 38, 2720-2737, 2010.

[33] Rasouli, S., Davvaz, B., Construction and spectral topology on hyperlattices, Mediterranean Journal of Mathematics 7, 249-262, 2010.

[34] Shahzamanian, M.H., Shirmohammadi, M., Davvaz, B., Roughness in Cayley graphs, Information Sciences 180, 3362-3372, 2010.

[35] Yamak, S., Kazanc1, O., Davvaz, B., Generalized lower and upper approximations in a ring, Information Sciences 180, 1759-1768, 2010.

[36] Yamak, S., Kazancı, O., Davaz, B., Approximations in a module by using set-valued homomorphisms, Int. J. Comput. Math. 88, 2901-2914, 2011.

[37] Zhao, B., Han, S.W., The ideal on hyperlattices, Fuzzy Systems and Mathematics 22, 44-51, 2008 . 